\title{
Gastric mixed adenoneuroendocrine carcinoma occurring 30 years after a gastroenterostomy
}

\author{
Mihaela Mădălina Gavrilescu ${ }^{1,2}$, Ionuț Huțanu ${ }^{*, 1,2}$, Ana-Maria Mușină ${ }^{1,2}$, Mihaela \\ Buna-Arvinte ${ }^{1,2}$, Ana Maria Patrașcu ${ }^{3}$, Iulian Radu ${ }^{1,2}$, Bogdan Filip ${ }^{1,2}$, Maria Gabriela \\ Aniței $^{1,2}$, Dragoș Viorel Scripcariu ${ }^{1,2}$, Viorel Scripcariu ${ }^{1,2}$
}

${ }^{1}$ 1st Surgical Unit, Regional Institute of Oncology, Iași, Romania; ${ }^{2}$ Department of Surgery, "Grigore T. Popa" University of Medicine and Pharmacy, Iași, Romania; ${ }^{3}$ Pathology Department, Regional Institute of Oncology, lași, Romania

\begin{abstract}
Gastroenteropancreatic neuroendocrine carcinomas (GEP-NENs) represent a heterogeneous group of rare tumors. The incidence of GEP-NENs has increased worldwide over the past decades, with the small intestine, rectum, and pancreas as the most common tumor locations. The epidemiological characteristics, pathogenesis and treatment have raised many questions, and some of them are still being debated. Here, we report a case of gastric collision tumor with large-cell neuroendocrine carcinoma and adenocarcinoma. A 73year-old male patient with a history of gastric resection performed 30 years ago, with no medical records revealing the type of resection or the reconstructive way, presented with epigastric pain. The endoscopy revealed a solid, ulcerated mass at the gastrojejunal anastomosis site from which a tissue biopsy was taken, which was reported as adenocarcinoma. For staging, the patient underwent an abdominal CT scan, which showed the thickening of the gastric wall adjacent to anastomosis and perilesional adenopathy. The patient underwent a subtotal gastrectomy and regional lymphadenectomy. A diagnosis of large-cell neuroendocrine carcinoma developed on the gastric stump associated with isolated foci of moderately differentiated tubular adenocarcinoma pT3N1G3 was made. Immunohistochemical analysis is essential for the diagnosis and classification of the lesion. To confirm the diagnosis, Chromogranin A and Synaptophysin are needed, and for prognostic evaluation the identification of $\mathrm{Ki}-67$ and mitotic figure count are required.
\end{abstract}

Keywords: neuroendocrine tumor; gastric carcinoma; adenoneuroendocrine carcinoma; G-NET; surgery.

\section{Introduction}

Gastroenteropancreatic neuroendocrine carcinomas (GEP-NENs) represent a heterogeneous group of rare tumors. Generally, the incidence of GEP-NENs has increased worldwide over the past decades, with the small intestine, rectum, and pancreas as the most common tumor locations [1].

Received: May 2018; Accepted after review: December 2018; Published: December 2018.

*Corresponding author: Ionuţ Huţanu MD, $1^{\text {st }}$ Surgical Unit, Regional Institute of Oncology, Iași, Romania.

Email: hionut65@yahoo.com
GEP-NENs were divided according to their site of origin into foregut (lung, stomach, duodenum and pancreas), midgut (distal jejunum, ileum, appendix, and cecum) and hindgut (colon and rectum) neuroendocrine tumors [2]. World Health Organization updates the classification and considers that GEPNETs represent tumors with different biological and clinical characteristics [3].

However, no improvement has been made in the clinical approach due to poor understanding of this pathology and a lack of a uniform, homogeneous classification. Quite recently, attention has again been directed to neuroendocrine tumors, so that in 2010 WHO 
proposed a new classification. According to this, GEP-NENs can be divided into NET G1, NET G2 or neuroendocrine carcinoma (NEC) G3. NET G1 and NET G2 are composed of well-differentiated tumor cells and have a Ki67 index $\leq 20 \%$, while NECs are poorly differentiated and have a proliferation index of over $20 \%[4,5]$.

Although the WHO classification is effective and has proven its usefulness over time, the European Neuroendocrine Tumor Society (ENETS) nevertheless considers it necessary to use the TNM classification for gastrointestinal neuroendocrine tumors in order to obtain a standardization of therapeutic management [3].

The epidemiological characteristics, pathogenesis and treatment have raised many questions, and some of them are still being debated.

Regarding the pathogenesis of NEC of the stomach, four pathways were reported based on their precursor cell-type: common-type adenocarcinoma; carcinoid tumor; multipotential stem cells and immature neuroendocrine cells. Recently, many NECs of the stomach are thought to originate from common-type adenocarcinoma. The endocrine cell carcinoma might have an intramucosal carcinoma origin. Differentiated tubular adenocarcinoma is thought to contribute to the occurrence of endocrine cell carcinoma [6].

\section{Case report}

A 73-year-old male patient with a history of appendectomy, adenomectomy and gastric resection performed 30 years ago, with no medical records revealing the type of resection or the reconstruction procedure, presented with asthenia, fatigue, weight loss, occasional epigastric pain and anemia. The endoscopy revealed a solid, ulcerated mass at the gastrojejunal anastomosis, from which a tissue sample was collected through biopsy. The histopathological report revealed gastric adenocarcinoma. For staging, the patient underwent a CT scan, which showed thickening of the gastric wall adjacent to the anastomosis and perilesional adenopathy (Figures 1 and 2).

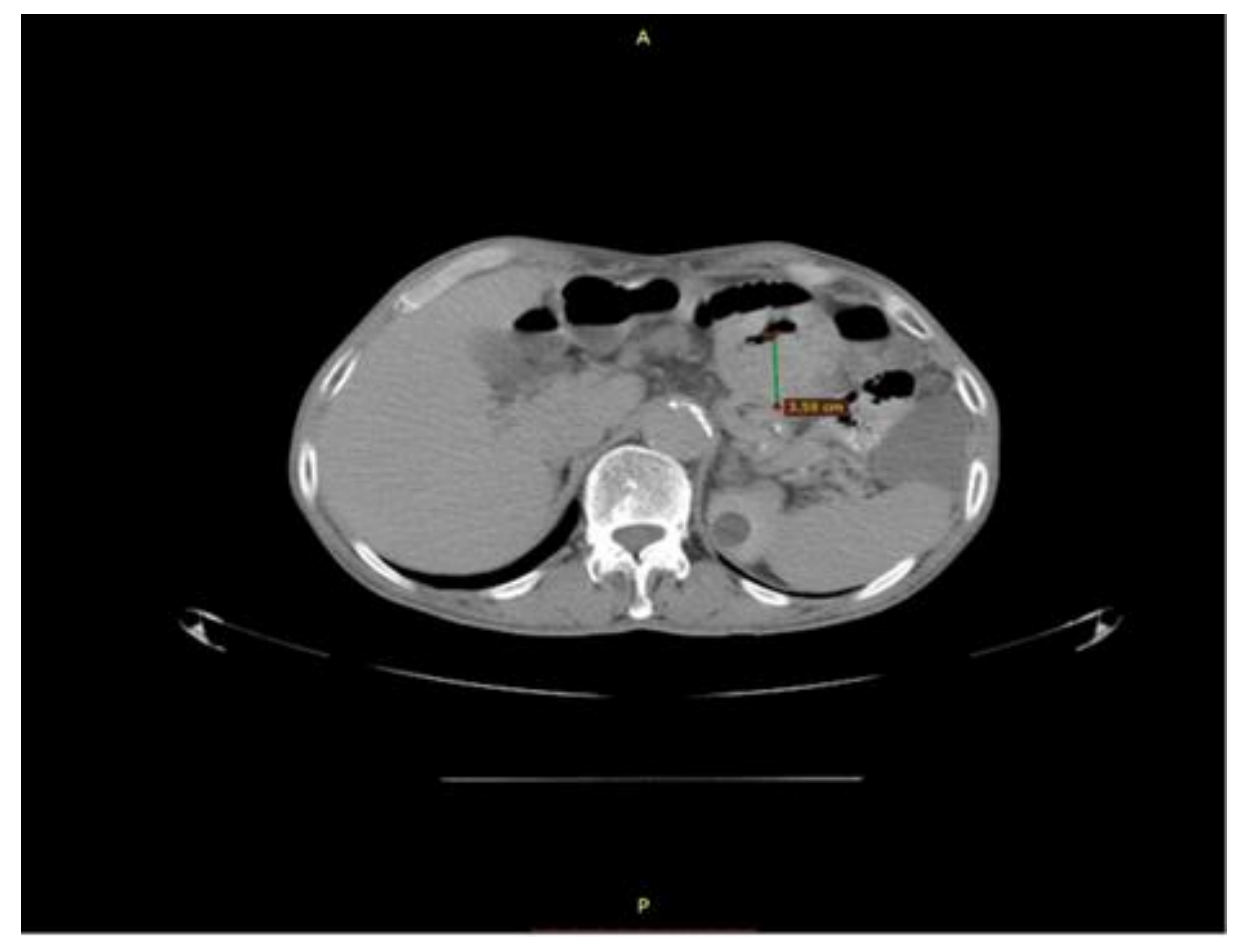

Fig. 1. CT-scan: Thickening of the gastric wall adjacent to anastomosis. 


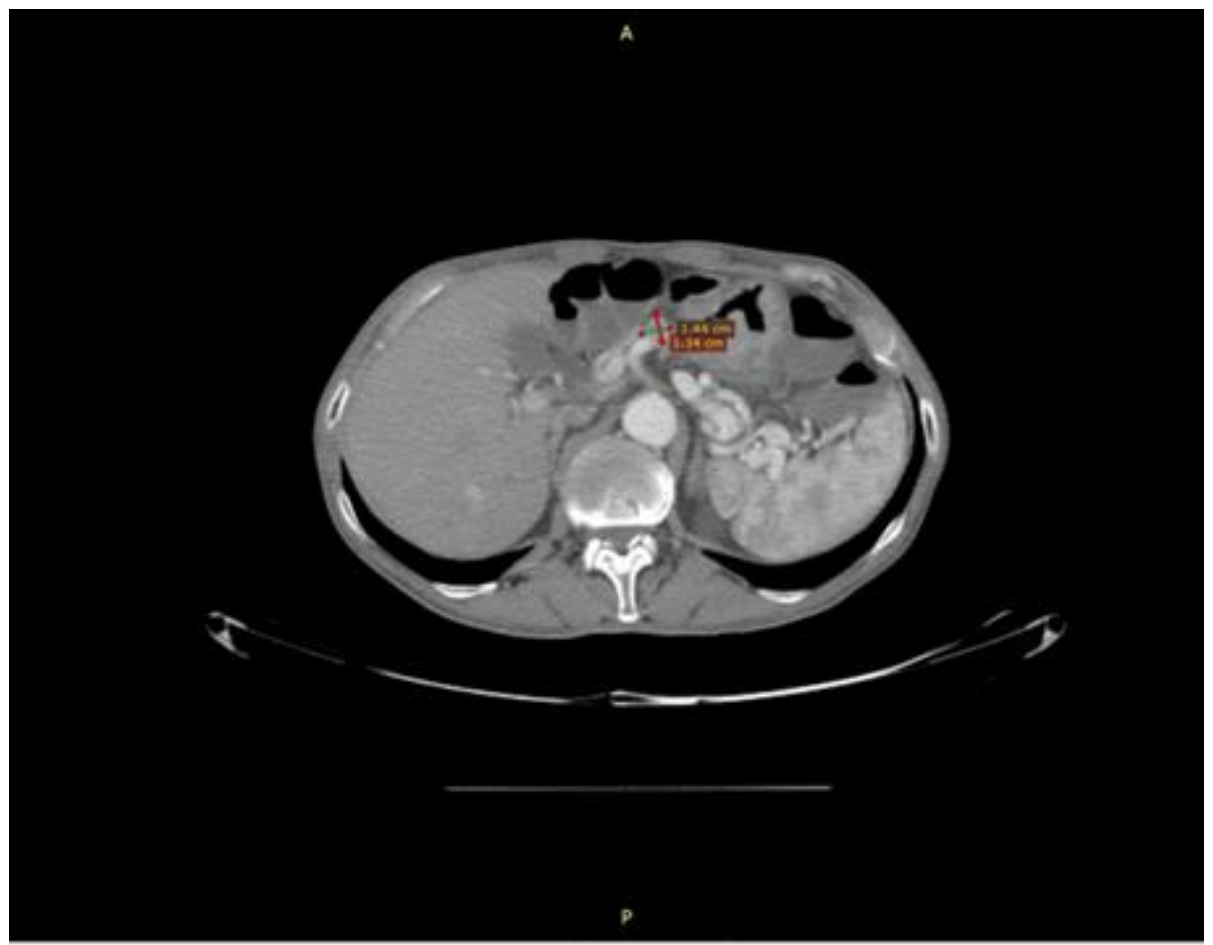

Fig. 2. CT-scan: Perilesional adenopathy.

On admission, laboratory findings revealed mild anemia $(\mathrm{Hb}=11 \mathrm{~g} / \mathrm{dl})$ with tumor markers within normal range. Laparotomy revealed a tumor mass at the level of gastrojejunal anastomosis and lymph node enlargement in the mesentery of the first jejunal loop, with no secondary tumors in the abdomen. Subtotal gastrectomy and regional lymphadenectomy with end-to-side Roux-en-Y gastrojejunal anastomosis were performed. The postoperative clinical course was favorable.

The pathology report described: a tumor with solid architecture, entirely invading the muscularis propria and extending into the perigastric adipose tissue without infiltration of the serosa; infiltration of the small intestine at the anastomosis site. The tumor periphery contained extremely rare tumor foci of moderately differentiated adenocarcinoma, with tubulo-glandular pattern, bordered by several layers of atypical columnar cells. Seven perigastric lymph nodes had no tumor infiltration, while 1 of 16 perijejunal lymph nodes showed metastasis, with areas of tumor necrosis. Immunohistochemistry showed that Synaptophysin and CD56 were positive in the solid tumor areas and negative in the tubuloglandular tumor foci (Figure 3); Chromogranin was positive in rare tumor cells, CDX2 was focally positive. A final diagnosis of large-cell neuroendocrine carcinoma developed on gastric stump associated with isolated foci of moderately differentiated tubular adenocarcinoma with a staging of pT3N1G3 was made.

The patient was referred to the oncologist for specialized treatment. He received adjuvant chemotherapy consisting of 6 cycles of cisplatin and etoposide.

One year after the surgery the patient showed no sign of recurrent disease.

\section{Discussions}

Mixed neuroendocrine carcinoma is a rare tumor of the digestive tract composed of two distinct tumor components in histological collision. By contrast, adenocarcinomas account for up to $95 \%$ of all gastric cancers and are by far the most studied tumors [7]. 

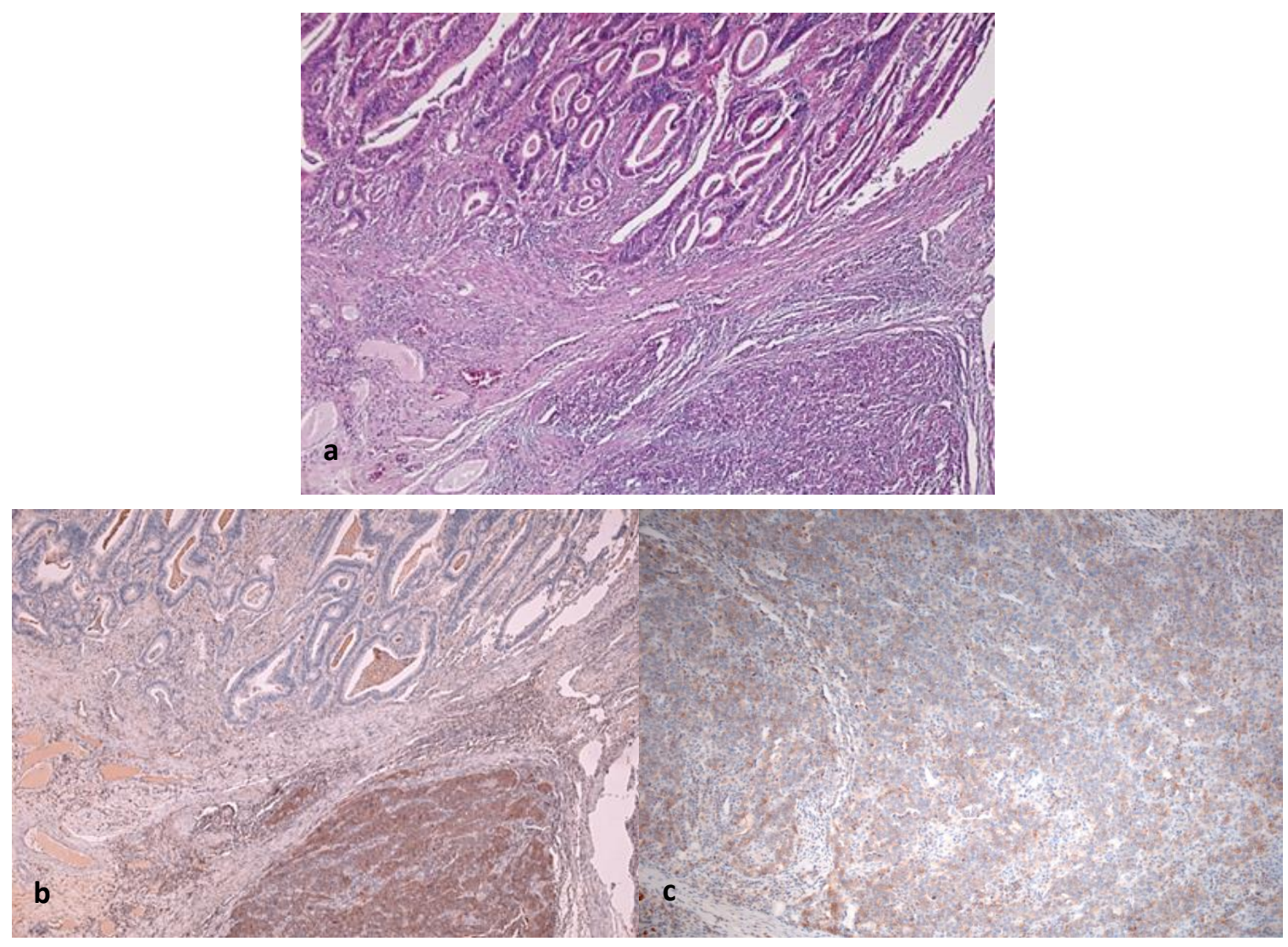

Fig. 3. (a): NEC and adenocarcinoma in juxtaposition (HE, x50). (b): Synaptophysin positive in neuroendocrine component, and negative in the adenocarcinoma component (IHC, anti-Synaptophysin Ab, x50). (c): CD56 positive in NEC (IHC, anti-CD56 Ab, x50).

Gastric resection for peptic ulcer is a wellknown risk factor for the development of gastric stump cancer. One theory attempting to explain this is based on the presence of bile reflux and chronic irritation of gastric mucosa by the pancreatic juice, both of them promoting and activating carcinogenesis [8].

We presented the case of a patient who underwent gastric resection for a benign condition 30 years ago and developed a mixed adenoneuroendocrine carcinoma (MANEC) at the site of anastomosis. During laparotomy we found a gastrojejunal anastomosis and we considered that first operation of this patient was a bulbar antrectomy, which was the standard indication for complicated peptic ulcer of the duodenum.

Gastric neuroendocrine carcinomas are very rare tumors that often coexist with an adenocarcinoma component. The definitive diagnosis of such a tumor is difficult to determine by endoscopic biopsy because the gastric mucosa is partially covered with adenocarcinoma, while neuroendocrine carcinomas develops in the submucosa and the other layers, making it difficult to evaluate through superficial tissue sampling [9]. The carcinogenesis of MANEC is unclear, although as much as $70.6 \%$ of gastric NEC cases include an adenocarcinoma component in the mucosa and/or submucosa. Given the more rapid growth pattern of NEC compared to adenocarcinoma and the fact that adenocarcinoma cells can have the same origin as NEC cells [10], it becomes clear that the lesion in question probably started out as a NEC which later developed multifocal adenocarcinoma. In terms of immunohistochemical assessment, the most commonly used markers are synaptophysin, Chromogranin and CD56, but other recently introduced markers such as $\mathrm{ASH} 1$ and NKX2.2 can be used. Also, a significant percentage of gastric neuroendocrine carcinomas express TTF-1 [9]. For prognostic evaluation, the identification of Ki-67 index and mitotic figure count are required. Other markers, such as p53, have been studied and 
correlated with the prognosis and the risk of metastasis [11].

Given the vast array of biomarkers needed for a definitive diagnosis, and keeping in mind that the majority of biopsies do not provide deep tissue samples [9], it becomes rather unlikely that an NEC diagnosis can be established before the operation itself is performed, especially when there are no symptoms pointing to anything more. Therefore, even though in our case the biopsy suggested adenocarcinoma, after resection and complex assessment of the surgical specimen a final diagnosis of mixed adenoneuroendocrine tumor was made.

In 2013, Ishida et al. published a study on a group of 51 patients diagnosed with gastric neuroendocrine carcinomas in which 41 curative treatment surgeries were performed. The authors reported that the overall 3- and 5years survival was $57.8 \%$ and $44.7 \%$, respectively [9]. Another study published in 2016 explored the clinic-pathological characteristics and surgical outcomes based on 135 consecutive patients with gastric neuroendocrine tumors (G-NETs). The median survival was not achieved for neither NET G1 nor NET G2 being by contrast 19 months (range 3-48) for NEC G3 and 10. 5 months (range 3-45) for MANEC [12].

G-NETs are divided into four types. Type 1 is a small, multifocal, polypoid tumor with a predilection for the gastric body and strong association with chronic autoimmune atrophic gastritis. On the other hand, type 2 G-NET, which is also multifocal, develops in association with multiple endocrine neoplasms type 1 (MEN-1), while type 3 is a sporadic tumor. Type 4 is represented by the rare neuroendocrine carcinoma [2]. We can surmise that survival rates of NETs and especially NEC (G3) are rather poor, even if small enough lesions could be resected endoscopically. In addition, the multifocality and tumor type variability further increase the

\section{References}

1. Rinzivillo M, Panzuto F, Delle Fave G. Controversies in the treatment of digestive neuroendocrine tumors. J Cancer Metasta Treat 2016; 2:304-309. therapeutic challenges. Both surgical margins and the staging according to the newly updated World Health Organization (WHO) classification are independent factors for oncological outcome after surgical resection, thus making follow-up steps more predictable [12].

\section{Conclusions}

Mixed tumor types are particularly difficult to accurately diagnose. Given the layering of adenocarcinoma on top of the deeper-growing neuroendocrine component, the usual diagnostic step of biopsy, which is paramount in orienting the therapy plan, often proves insufficient, as in our case.

The particularity of the case is based on the presentation 30 years after a gastric resection for peptic ulcer of a tumor initially diagnosed as adenocarcinoma. After resection and complex assessment of the surgical specimen, a final diagnosis of mixed adenoneuroendocrine tumor was made. Our patient benefited from a lymphadenectomy limited to the perigastric nodes and those along the superior mesenteric vessels due to the particularities of lymphatic drainage in a gastrojejunal anastomosis. Nevertheless, only one of the 23 lymph nodes was positive. The patient was referred to the oncologist for specialized treatment and one year after surgery the patient is negative for disease.

\section{Consent}

Written informed consent was obtained from the patient for publication of this case report.

\section{Competing interests}

The authors declare that they have no competing interests.

2. Schott $M$, Klöppel G, Raffel $A$, Saleh $A$, Knoefel WT, Scherbaum WA. Neuroendocrine neoplasms of the gastrointestinal tract. Dtsch Arztebl Int 2011; 108(18):305-312. 
3. Rindi G, Klöppel G, Alhman H, et al. TNM staging of foregut (neuro)endocrine tumors: a consensus proposal including a grading system. Virchows Arch 2006; 449(4):395-401.

4. Bosman FT, Carneiro F, Hruban $\mathrm{RH}$, Theise ND. WHO classification of tumours of the digestive system, $4^{\text {th }}$ Edition. Lyon: IARC, 2010.

5. Hijioka S, Hosoda W, Mizuno N, et al. Does the WHO 2010 classification of pancreatic neuroendocrine neoplasms accurately characterize pancreatic neuroendocrine carcinomas? J Gastroenterol 2015; 50(5):564572.

6. Aoyagi K, Kizaki J, Isobe T, Akagi Y. A case of gastric cancer with neuroendocrine carcinoma, signet ring cell carcinoma components, and intramural metastases. Am J Case Rep 2016; 17:274-279.

7. Düzköylü $Y$, Aras O, Bostancı EB, Keklik Temuçin T, Ulaş M. Mixed adenoneuroendocrine carcinoma; case series of ten patients with review of the literature. Balkan Med J 2018; 35(3):263-267.

8. Sitarz R, Maciejewski R, Polkowski WP, Offerhaus GJ. Gastoroenterostoma after Billroth anterectomy as a premalignant condition. World J Gastroenterol 2012; 18(25):3201-3206.

9. Ishida $M$, Sekine $S$, Fukagawa $T$, et al. Neuroendocrine carcinoma of the stomach morphologic and immunohistochemical characteristics and prognosis. Am J Surg Pathol 2013; 37(7):949-959.

10. Fukuba NY, Takafumi I, Shunji S, et al. Gastric mixed adenoneuroendocrine carcinoma with a good prognosis. Inter med 2014; 53:25852588.

11. Dias AR, Azevedo BC, Alban LBV, et al. Gastric neuroendocrine tumor: review and update. Arq Bras Cir Dig 2017; 30(2):150-154.

12. Shen $C$, Chen $H$, Chen $H$, et al. Surgical treatment and prognosis of gastric neuroendocrine neoplasms: a single-center experience. BMC Gastroenterol 2016; 16:111. 